\title{
Moderate Intra-abdominal Free Fluid on Computed Tomogram in Patients with Blunt Trauma Abdomen: Time to Shun Non-operative Approach
}

\author{
Debajyoti Mohanty • Ashwani Kumar Dalal • \\ Pankaj Kumar Garg
}

Published online: 15 August 2014

(C) Société Internationale de Chirurgie 2014

We have read with interest the article by Mahmood et al. [1] on the significance of detection of intra-abdominal free fluid sans solid organ injury on computed tomogram (CT) in hemodynamically stable patients with blunt trauma abdomen (BTA). Their retrospective analysis of 122 cases reaffirms the importance of thorough and repeated clinical examination in the decision-making process for laparotomy in patients with mesenteric and bowel injury.

The authors applied multivariate logistic regression analysis for identifying the predictors associated with therapeutic laparotomy. Odds ratio (OR) was calculated for the risk factors to ascertain their relationship with therapeutic laparotomy. The OR is a measure of association between an exposure of interest and a certain outcome. In other words, it is a ratio between the odds that a particular exposure will result in an outcome to the odds that the same outcome will result in absence of that exposure. Apart from identifying the exposure as a potential risk factor for a defined outcome, the OR also determines the magnitude of effect of that particular exposure on the outcome. The exposure is said to have no effect on the outcome when the OR is one. A value greater than one suggests a positive association between the exposure and the outcome, whereas a value less than one suggests a negative association between them. In the study by Mahmood et al. age, mechanism of injury, and presence of seat belt sign were found to have statistically significant positive association with therapeutic laparotomy following calculation of OR;

D. Mohanty $(\bowtie) \cdot$ A. K. Dalal · P. K. Garg

Department of General Surgery, All India Institute of Medical

Sciences, Raipur, Chhattisgarh 492099, India

e-mail: debajyoti.mohanty@gmail.com

\section{P. K. Garg}

Department of Surgery, University College of Medical Sciences and Guru Teg Bahadur Hospital, Delhi 110095, India however, two of these predictors had very wide confidence intervals, implying very low precision of the results.

The logistic regression table outlining the predictors for therapeutic laparotomy depicts significant $p$ value with a high OR and a wide confidence interval for the mild amount of abdominal fluid evident on CT scan. We think the presented data points show otherwise, in that a moderate rather than a mild amount of free fluid on CT scan has a stronger association with therapeutic laparotomy. The logistic regression analysis for a moderate amount of free fluid on CT scan as a predictor for therapeutic laparotomy revealed an OR of $8.66(p=0.0001)$ and a CI at 3.03-24.7 (statpages.org/logistic.html). In light of the above observations, a moderate amount of free fluid on CT of the abdomen should have been considered a strong predictor for therapeutic laparotomy. The authors overlooked this fact and concluded that CT scans are inaccurate for the prediction of bowel injury and the need for laparotomy. They have also advocated against immediate celiotomy in patients with abdominal injury. We think their opinion is nonspecific and not in sync with the objectives and the results of their study.

While the role of clinical examination in deciding the need for laparotomy in patients with BTA is undisputed, detection of moderate free fluid on CT scan should tilt the balance in favor of laparotomy in patients with equivocal findings on clinical examination of the abdomen.

\section{References}

1. Mahmood I, Abdelrahman Y, Siddiuqqi T et al (2014) Significance of computed tomography finding of intra-abdominal free fluid without solid organ injury after blunt abdominal trauma: time for laparotomy on demand. World J Surg 38:1411-1415. doi:10.1007/ s00268-013-2427-5 